\title{
Eutectic and peritectic solidification patterns
}

\author{
Silvère Akamatsu ${ }^{\mathrm{a}}$, Mathis Plapp ${ }^{\mathrm{b}, *}$ \\ ${ }^{a}$ Sorbonne Universités, UPMC Univ. Paris 06, CNRS-UMR 7588, Institut des \\ NanoSciences de Paris, F-75005, Paris, France \\ ${ }^{b}$ Condensed Matter Physics, Ecole Polytechnique, CNRS, 91128 Palaiseau, France
}

\begin{abstract}
Recent advances in the understanding of eutectic and peritectic two-phase pattern formation under purely diffusive transport are reviewed. The parallel progress of two key techniques, namely, in-situ experimentation with model, low-melting transparent and metallic alloys in thin and bulk samples, and numerical phase-field simulations, is highlighted. Experiments and simulations are interpreted in the light of the theory of non-equilibrium pattern formation phenomena. Focus is put on microstructure selection and morphological transitions, multiscale patterns in ternary alloys, and the influence of crystallographic effects on pattern formation. Open problems, for example on crystallographic effects, irregular eutectics, and peritectic solidification, are outlined.
\end{abstract}

Keywords: solidification, eutectic alloys, peritectic alloys, in situ experiments, phase-field modelling

\section{Introduction}

The solidification of eutectic and peritectic alloys of composition close to a nonvariant point in the phase diagram frequently produces multi-phased microstructures in the bulk. Eutectic or peritectic structures are also frequently found to form during the late stages of a solidification process. Those microstructures are essentially a frozen-in trace of the pattern-formation dynamics at the solidification front $[1,2,3]$. The latter is governed by the

\footnotetext{
${ }^{*}$ Corresponding author

Email addresses: silvere.akamatsu@insp.jussieu.fr (Silvère Akamatsu), mathis.plapp@polytechnique.fr (Mathis Plapp)
} 
interplay between solute diffusion in the liquid and the response of the interfaces due to capillary and kinetic effects. We will focus on metallic alloys and organic "metal analogs", for which the solid-liquid interfaces are nonfaceted and kinetic effects can be neglected; irregular eutectics will therefore not be addressed here. We will concentrate on recent developments in this field; earlier works are covered in textbooks $[4,5]$ and previous review articles $[6,7,8,9,10,11]$.

In recent years, considerable progress on complex morphological features of multiphased solidification microstructures has been made on a fundamental level thanks to the parallel advancement of in-situ experimentation and numerical simulations. The rapid development of phase-field modelling $[12,13]$ makes it now possible to perform quantitative simulations of multiphase solidification in three dimensions for extended systems $[14,15,16]$. Experimentally, in addition to major improvements of the classic thin-sample directional solidification technique, novel real-time observation methods have been developed, which allow for the imaging of eutectic front patterns in bulk samples of transparent organic alloys with a micron-scale resolution $[17,18]$. Moreover, implementing high-resolution X-ray radiography and tomography has brought unprecedented information on solidification microstructures in bulk and thin metallic samples [19].

Theoretical interpretation of the data obtained from experiments and simulations crucially benefits from the concepts and methods of fundamental physics of nonequilibrium pattern formation. The interaction of this field with solidification science started in the 1980's [20] and has been fruitfully pursued since then, thus shedding new light on increasingly complex questions that include microstructure selection and morphological transitions, solute redistribution transients, multiscale patterns in ternary alloys and interfacial-anisotropy (crystallographic) effects. Therefore, in the following, we will seek to establish a close link between experimental and numerical results, and the theory of pattern formation.

To this end, it is useful to concentrate on model (binary and ternary) alloys and investigate them in well-controlled laboratory experiments. In particular, the use of thin samples or microgravity environments can suppress the convective motions in the liquid. This simplifies the numerical treatment, and makes the results directly comparable to theories of diffusionlimited crystal growth. While this approach omits several aspects that are important for practical applications (multiple components, convection), it can validate the theories and models that are needed as a basis for a com- 
plete understanding of such situations. Most of the results that are reviewed below have been obtained either by (in-situ) directional-solidification experiments, that is, solidification in a fixed temperature gradient of magnitude $G$, with an imposed velocity $V$, or by phase-field simulations.

In the following, we will discuss subsequently binary eutectics, with an emphasis on various phenomena that influence the dynamics of two-phase pattern formation, ternary eutectics, in which an entirely new microstructure, namely spiral two-phase dendrites, was discovered, and peritectics. The review will be concluded by a list of what we consider to be important open questions.

\section{Binary eutectics}

\subsection{Background}

In a binary eutectic alloy, two distinct solid phases, generically called $\alpha$ and $\beta$ in the following, coexist with the liquid phase at the eutectic temperature $T_{E}$, with the composition $C_{E}$ of the liquid falling in between the compositions of the two solids. For a range of compositions around $C_{E}$, the liquid can solidify into a two-phase composite (coupled growth). For two solid phases with isotropic and nonfaceted solid-liquid interfaces, the theoretical description of this growth mode includes bulk diffusion in the liquid phase, mass conservation at the moving interfaces (Stefan condition), and local equilibrium at interfaces (Gibbs-Thomson equation) and trijunction points (Young's law). The resulting free-boundary problem has been stated in many publications, and can be found for example in Ref. [21].

The cornerstone for our understanding of eutectic growth fronts is the Jackson-Hunt theory [3] (also see the pioneering works by Zener and Hillert $[1,2]$ ), which solves an approximate version of this problem for a steady periodic pattern of spacing (spatial period) $\lambda$. The interplay between diffusion and capillarity is characterized by a scaling length $\lambda_{m}$ (at which the front undercooling exhibits a minimum in directional solidification), which is proportional to $V^{-1 / 2}$. Eutectic-growth dynamics essentially depends on a single parameter, $\lambda / \lambda_{m}$, proportional to $\lambda V^{1 / 2}$, and is little sensitive to the magnitude of the temperature gradient $G[22]$.

The two morphologies that are most frequently observed in eutectic composites are parallel platelets (lamellae) of the two phases, and fibers (rods) of one phase, located on the nodes of a regular triangular lattice, and surrounded by a matrix of the other phase. Jackson and Hunt have calculated 
$\lambda_{m}$ for these two morphologies. In fact, many other arrangements of the solid phases along the (planar) front are also observed. This morphological "multistability" is a direct consequence of the symmetry properties of the system. The same symmetry elements are also exhibited by many other pattern-forming systems with an axial symmetry, such as Rayleigh-Bénard convection, Faraday surface waves, reaction-diffusion systems, and magnetic thin films. Lamellae and fibers in eutectics correspond to regular stripe and dot patterns that are common in these systems. For the present review, it is useful to take advantage of the knowledge accumulated on generic aspects of these systems in the theories of nonequilibrium pattern formation [23, 24].

In nonequilibrium pattern forming systems, steady periodic patterns are generally stable over a finite range of values of the spatial period $\lambda$, at fixed control parameters. Inside the region of stability in the space of control parameters (stability balloon), pattern uniformization is ensured by a generic relaxation mechanism of diffusive nature ("phase" or "spacing" diffusion). The limits of the stability balloon are set by morphological instabilities (bifurcations), which break one or several symmetries of the underlying regular pattern. Outside the basic-state stability balloon, the system restabilizes into a steady, symmetry-broken periodic pattern or displays a complex spatiotemporal dynamics.

These facts imply that in laboratory experiments, particular attention has to be paid to the preparation of the initial state: which kind of pattern forms depends, in practice, on sample history and boundary conditions. This has been explicitly demonstrated for lamellar patterns in a thin-sample geometry [25]. A common directional-solidification protocol (also see numerical simulations [26]) consists of establishing a steady-state pattern at constant $V$, and observing the response of the system to successive $V$ jumps.

In this way, many results have been obtained in thin-sample directional solidification of transparent alloys. The stability balloon (stable spacings as a function of alloy concentration) has been determined experimentally and numerically. Its limits are set by various oscillatory instabilities for large spacings, and by lamella elimination for small spacings $[25,26,27]$. This constitutes a reference basis for more recent studies on bulk solidification, which are reviewed in the next section. Bulk solidification is a much more challenging subject, theoretically, due to the large number of geometrical degrees of freedom (two-dimensional translational and rotational symmetry) of the ("ideal") system, and experimentally. New real-time experimental methods have been set up, which combine the use of a long-distance opti- 
cal microscope with subsequent image processing for yielding the equivalent of a "top view" of the growth front in samples of inner thickness reaching $1 \mathrm{~mm}[17,18]$. Due to possible thermo-solutal instabilites, experiments are mostly limited to near-eutectic compositions. Efficient and accurate phasefield models for bulk eutectic growth have been developed [28] that have made it possible to perform three-dimensional simulations on a length scale of several microstructural units $[14,15]$.

In the following, we will review the morphological transformations of lamellar and rod eutectics, as well as space-time dynamical features and topological defects which determine the long-time evolution of extended patterns in directional solidification. Finally, the universality of the above-mentioned phenomenology breaks down in more "realistic" situations, when new physical effects (external or internal) break the symmetries of the ideal system. We will consider two dynamic-forcing phenomena: the effect of an imperfectlyshaped temperature gradient, and crystallographic effects.

\subsection{Dynamics of patterns with isotropic interfaces}

Lamellar eutectics. Lamellar eutectic patterns in bulk-sample solidification have been studied both in experiments and simulations $[29,30,14]$. A new instability mode was identified in the experiments, namely, the zigzag instability (Fig. 1a), which is a transition from straight to chevron patterns or wavy lamellae (for general bases of the zigzag bifurcation, see Ref. [23]). Phase-field simulations in three dimensions [14] have helped to clarify the connection to the results obtained in thin samples $[25,26]$. Other instability modes, that extend the known two-dimensional modes to three dimensions, do exist but grow more slowly than the zigzag instability for all parameter sets that were investigated. This explains why in the experiments only the zigzag mode was clearly identified.

Let us now turn to the dynamics of extended systems. In the experiments that revealed the existence of the zigzag instability in lamellar eutectics [29], quite frequently the eutectic fronts did not self-organize into well-ordered lamellar arrays, but remained disordered during the entire time of the experiment (Fig. 1b): while the characteristic spacing is clearly visible, the local orientation of the lamellae varies from point to point in space, and no global ordering emerges. In the simulations, this is actually the generic behavior when a mixture of the two phases with random arrangement is used as a starting guess (Fig. 1d). After rapid formation, the evolution of such "labyrinth" patterns slows dramatically down, and an ordered lamellar state 


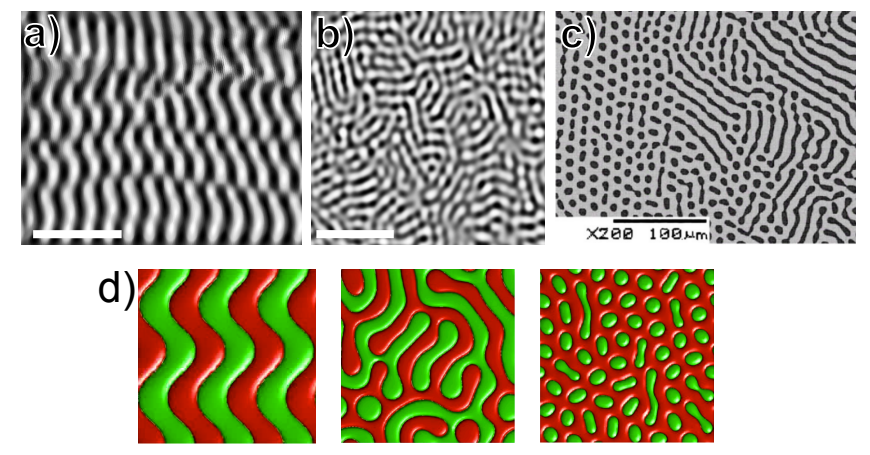

Figure 1: Eutectic growth patterns in bulk directional solidification. Top-view observations (transparent eutectic alloy $\mathrm{CBr}_{4}-\mathrm{C}_{2} \mathrm{Cl}_{6}$, see Ref. [17]): a) zizag pattern $\left(V=0.39 \mu m s^{-1}\right.$; note the presence of "phase jumps", reminiscent of the "fault lines" in metallic ingots); b) "labyrinth" pattern $\left(V=1.0 \mu \mathrm{ms}^{-1}\right)$. c) Transverse section of a directionally solidified Al$41 \mathrm{wt} . \% \mathrm{Cu}$ alloy (from [31] with permission), which illustrates coexistence between rods, elongated rods, and lamellae. Bars: $100 \mu \mathrm{m}$. d) Three-dimensional phase-field calculations performed for a eutectic alloy with symmetric phase diagram [14, 15].

is not reached even in the longest experiments/simulations [15]. Generally speaking, it is known for stripe patterns in various other systems that ordering, if it occurs at all, is extremely slow in extended isotropic systems [24]. Labyrinth patterns in eutectic growth is a signature of the absence of a strong interfacial-anisotropy effect.

In principle, the symmetry-breaking instabilities discussed so far conserve the average spacing (that is, the total number of lamellae or rods). There are other instabilities, in particular, elimination and branching, which increase or decrease the spacing, respectively, and can thus bring the system back into the stability balloon. A lamellar branching mechanism has been clearly evidenced in an experimental directional-solidification study of a lamellareutectic $\mathrm{Al}-\mathrm{Al}_{2} \mathrm{Cu}$ alloy [32], during which the pulling velocity was suddenly increased by a factor of four. The instability of lamella elimination in bulk systems has not been addressed in detail. It is important to note that both lamellar branching and elimination also generally introduce a strong topological disorder in the form of lamella terminations into the pattern. Both experiments and simulations indicate that this disorder is persistent over the accessible time scales.

Finally, it is worth mentioning that the problem of the fault lines that are present in large density in cross-sections of bulk lamellar-eutectic materials still remains largely open. More precisely, the dynamic mechanism that 
leads to their formation has not been firmly established (see Ref. [29] and Fig. 1a), and the proposition that fault lines are stabilized by crystal lattice defects (subboundaries) has not been definitely confirmed [33]. To our best knowledge, there are no numerical simulations available of that phenomenon.

Rod-like eutectics. Rod eutectics tend to form when the volume fractions of the two phases are strongly different. They generally organize into small hexagonal-stacking domains. In-situ experimental measurements indicate that rod eutectic patterns are stable over a finite interval of rod-spacing values limited, in practice, by an elimination (lower limit) and a branching (upper limit) instability (the latter being close to $\lambda_{m}$ ) $[34,35]$. However, more complex features have been revealed both experimentally and numerically. First, for moderately assymmetric volume fractions (minority phase of the order 20 to 30 percents), a shape instabiity was observed in phase-field simulations [15]: above a critical spacing, rods elongate, and become dumbbellor peanut-shaped for even larger spacings, with the axis of elongation being directed toward the first or the second neighbors in the triangular rod lattice, respectively, depending on initial and boundary conditions. Experiments show that elongated-rod patterns are very sensitive to distorsions in the rod lattice [31]. Elongated rods have also been frequently observed during transient (precursory to rod splitting) [35], as part of disordered patterns [32, 31, 36], see Fig. 1c, or in strongly confined geometries [37].

For lower volume fractions of the rod phase, an oscillatory dynamics has been observed both in phase-field simulations and real-time experiments [35]. For symmetry reasons, collective oscillations similar to the ones of hexagonal deep-cell patterns in directionally solidified dilute binary alloys [38, 39] could be expected. However, since the rod oscillations were observed in highly disordered patterns, no clear symmetry could be identified.

Lamella-to-rod transition. Interesting results have also been obtained on the transition from lamellae to rods and vice versa. Both in experiments [31] and simulations [15], it was found that this transition is not sharp, but occurs over an extended range of alloy concentrations. In the simulations, the transition can be triggered on purpose by changing the composition of the liquid ahead of the eutectic front with time, and a hysteresis was observed: the transition between lamellae and rods does not occur at the same composition in the "forward" and "backward" directions. In experiments, composition variations along the front are often created by convection, so that lamellae, rods, and mixed morphologies can be simultaneously observed in the same sample $[31,40]$. It it worth mentioning that a well-controlled 
study of the lamella-to-rod transition might be possible in a microgravity environment, in which composition gradients in a liquid can be maintained over sufficiently long times because of the absence of natural convection [41].

\subsection{Effect of misaligned and curved isotherms}

Slight deformations of the thermal field are difficult to avoid experimentally, and can have a determining "selection" effect during eutectic growth. First, because of an asymmetry of the setup, the isotherms, instead of being strictly perpendicular to the main growth axis, can be slightly misaligned. This thermal bias imposes a global drift of the pattern along the slanted isotherm. In transparent-alloy experiments with isotherms exhibiting a (tunable) bias angle, a regular pattern generated close to one of the walls propagated laterally and enventually "invaded" the entire cross-section [16]. This entails the formation of regular (straight or zigzag) lamellar eutectics at the expense of a labyrinth pattern within a time lapse accessible in the experiments. This process has been reproduced in numerical simulations.

Second, a non-trivial effect of a macroscopic deformation of the isotherms due to different heat conductivities of the solid, the liquid, and the sample walls has been observed on rod patterns in a transperent alloy. Since the rods grow approximately perpendicular to the large-scale front envelope, a slight convex curvature of the isotherms produces a continuous stretching of the pattern. Thus, the average spacing continuously increases, until the stretching effect is globally balanced by the creation of new rods by splitting. After a long solidification time, the statistical spacing distribution was measured to be independent of initial conditions. A forcing (here, by the curvature of the isotherms) thus drives the system to operate, on average, in the vicinity of a marginal stability point (here, the rod splitting threshold). This observation possibly provides an answer to the question of the "selection" of the eutectic spacing [34]. This process has, to our knowledge, not yet been explored in simulations. Moreover, long experiments without rod splitting nor elimination could be achieved by gradually increasing $V$ with time (also see $[42])$.

\subsection{Anisotropic interphase boundaries}

In the modern analyses of eutectic solidification that we have reviewed above, crystallographic effects were left aside. However, it has been known since a long time that eutectic patterns can be dramatically influenced by crystallography: in a single sample, patterns usually differ between different 
eutectic grains. In a (eutectic) grain, the two phases that form the composite solid both have a fixed lattice orientation. Therefore, the free energy of the interphase boundary between the two solids, as well as its anisotropy, are fixed within a given grain, but vary between different grains [43]. In nonfaceted binary eutectic alloys which present at least one special, or epitaxial, orientation relationship (OR) between the two solid phases, there exist one or several families of coincidence planes for the interphase boundaries, which correspond to deep minima of the interphase boundary free energy as a function of its inclination. In special-OR grains, the eutectic growth dynamics is dramatically altered. In particular, growth microstructures with tilted lamellae are often observed: the interphase boundary remains "locked" onto a low-energy direction. In contrast, in grains without special OR, the lamellae follow the direction of the thermal gradient ("floating" grains). The difference between locked and floating grains is illustrated in Fig. 2, where it can also be seen that the spacing remains inhomegenous in locked grains.

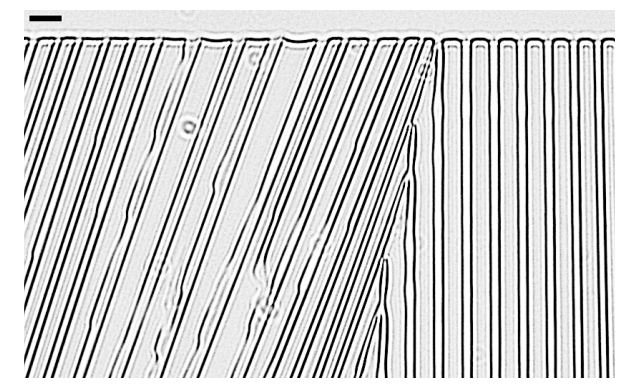

Figure 2: Locked (on the left) and floating (on the right) eutectic grains during thin-sample directional solidification $\left(\mathrm{CBr}_{4}-\mathrm{C}_{2} \mathrm{Cl}_{6} ; V=1.0 \mu m s^{-1}\right)$. Bar: $20 \mu m$.

The influence of interphase boundary anisotropy on lamellar eutectic growth was recently analyzed in a series of papers [44, 45, 46]. Experiments indicate that the anisotropy of nonfaceted solid-liquid interfaces is weak (as is to be expected), and can therefore by neglected in the analysis. The anisotropy of the (solid-solid) interphase boundary enters the problem of eutectic growth only at one place: in the local equilibrium condition at the trijunctions. Here, Young's law has to be replaced by the Young-Herring condition. Experiments suggest that the solid-liquid interfaces retain an approximately symmetric shape with respect to the mid-plane between two neighboring trijunctions, even if the lamellae are tilted. This yields a condition for the contact angles at the trijunction, which makes it possible to 
determine the growth direction if the anisotropy of the interphase boundary energy is known [44]. Within the domain of application of this symmetric pattern approximation (SPA), quantitative information on the anisotropy (Wulff plot) of interphase boundary energies from experiments in the rotating directional solidification setup [45] in which the sample can be rotated with respect to the temperature gradient. The SPA conjecture was tested by two-dimensional numerical simulations using both phase-field and boundaryintegral methods [46]. A good agreement between simulated and calculated tilt angles was found, and the dynamic lamellar-locking effect confirmed.

\section{Ternary eutectics}

\subsection{Eutectic cells and dendrites}

The solidification of ternary eutectic alloys delivers complex, multiscale microstructures within which one-, two- and three-phased regions can coexist in the solid, depending on both the composition of the melt and growth conditions [47]. We consider first two-phased eutectic growth, that is, univariant solidification along a eutectic valley in the ternary phase diagram, which typically occurs when a relatively small amount of a third compound is added to a binary eutectic alloy of reference. The third component is rejected by the two-phased solid, and diffuses over long distances in the liquid, which makes possible the development of fingering instabilities of the composite growth front. Important questions concern, in particular, (i) the coupling of the large-scale cellulation and the underlying eutectic growth, (ii) the existence of steady-state dendrites, and (iii) the influence of an interfacial anisotropy.

Eutectic cells. Eutectic cells arise from a Mullins-Sekerka instability of the planar coupled-growth front on a scale much larger than the eutectic spacing $\lambda$, for pulling velocities above a given threshold value $V_{c}$ [48]. The value of $V_{c}$ can be basically estimated by a constitutional-supercooling criterion, but eutectic cells actually exhibit an additional complexity due to a nonlinear interaction between the modulations of the front shape and the eutectic growth dynamics $[49,50,51]$. In practice, however, deep eutectic cells do not emerge from a linearly unstable mode, but rather via the mediation of local structures, in particular the so-called eutectic finger (see below). Once formed, eutectic cells form unsteady patterns, with frequent branching and elimination events forced by the curved eutectic-cell profile. Similar features are apparent in cross-section metallographs in metallic alloys [52], and have been recently observed in situ in bulk transparent alloys [18]. In 
systems that exhibit strong interfacial anisotropy effects, the shape and the stability of eutectic cells can depend markedly on the crystal orientation of the eutectic grain $[53,33]$.

Spiral two-phase dendrites. Solidification of ternary eutectic alloys containing a large amount of the third component leads to the formation of highly branched structures and to eutectic dendrites, that is, needle-shaped crystal growth morphologies with a regular two-phase internal structure. Few studies have so far been devoted to these objects $[48,54]$. Recently, a novel ternary-eutectic growth shape called spiral(ing) two-phased dendrite (in short, spiral dendrite) has been discovered and characterized during directional solidification of a nonfaceted transparent alloy [55], see Fig. 3a. Like a one-phased dendrite, a spiral dendrite possesses a paraboloidal outer shape with sidebranches. Its key feature is a spiraling eutectic growth pattern located at the tip, which permits continuous eutectic growth along the curved solid-liquid interface. A duplex helicoidal microstructure is thus delivered in the solid. It was observed that the growth direction of spiral dendrites could change during short transients. The tip radius of curvature $\rho$ and the eutectic spacing $\lambda$ were measured as a function of the growth velocity $V$. In brief, $\rho$ and $\lambda$ were found to be very close to each other, and to the minimum undercooling spacing $\lambda_{m}$ of the binary eutectic alloy of reference. This indicates a strong morphological selection of spiral dendrites, with $\rho \sim \lambda \sim V^{-1 / 2}$, independently of interfacial anisotropy. This scaling behavior has been established on the basis of Ivantsov's theory of the needle-crystal growth and the Jackson-Hunt theory of eutectic growth [56]. Spiral dendrites have been recently reproduced by numerical simulations with a phase-field model that permits efficient computation of large, multiscale systems [57, 58], see Fig. 3b. This study mostly confirms the above conclusions, but reports that a certain amount of kinetic anisotropy was needed for stabilizing spiral dendrites, in apparent contradiction with experiments. Clear experimental evidence of spiral dendrites in metallic alloys is still lacking.

As mentioned above, another kind of two-phase fingers, which consist of two symmetrically disposed fingers of one solid phase separated by a thin crystal of another solid phase, have also been observed in transparent eutectic alloys with a dilute impurity. The growth direction of a two-phase finger is free. In contrast with the spiral dendrite, eutectic growth occurs solely at the tip of two-phase fingers, which are favored by an off-eutectic composition, and a two-dimensional geometry. According to a recent theoretical analysis, twophase fingers could exist in binary eutectic alloys, but for undercooling values 


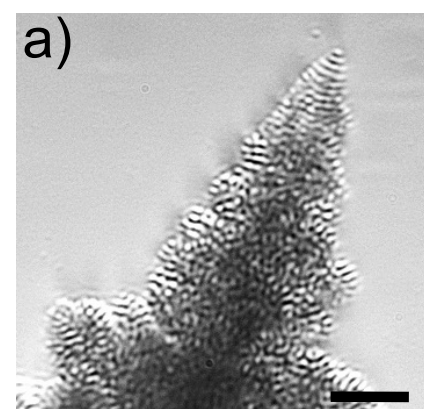

b)

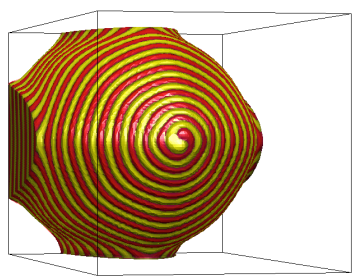

Figure 3: Spiral two-phase dendrites. a) Real-time observation (side view) during directional solidfication of a transparent ternary alloy (see Ref. [55]). Bar: $20 \mu \mathrm{m}$. b) Phase-field simulation (courtesy of the authors of Ref. [57]).

much larger than those usually reached in lamellar patterns in directional solidification [59].

\subsection{Three-phase growth}

Various three-phased microstructures are produced during nonvariant solidification of ternary eutectic alloys of composition close to a eutectic point (intersection of three eutectic valleys in the phase diagram). In such systems, coupled-growth front patterns involve three eutectic solids, and can exhibit many different tiling motifs. This complex problem still remains poorly addressed in the metallurgic litterature. Three-phased lamellar growth patterns in a two-dimensional directional-solidification geometry have been recently considered. Let us generically call $\mathrm{A}, \mathrm{B}$ and $\mathrm{C}$ the three eutectic phases, and note $\mathrm{ABC}, \mathrm{ABAC}, \mathrm{ABABAC}$, etc, the stacking motif in a periodic pattern. A theoretical analysis à la Jackson-Hunt has been performed, leading to the standard definition of a repeat-unit dependent minimum-undercooling spacing $\lambda_{J H}$, proportional to $V^{-1 / 2}$, as a relevant scaling quantity [60, 61]. This scaling behavior has been confirmed experimentally for lamellar patterns with an ABAC motif (the median planes of the $\mathrm{B}$ and $\mathrm{C}$ lamellae are mirror symmetry planes) during thin-sample directional solidification of two different low-melting ternay eutectic alloys - the metallic $\mathrm{In}_{-} \mathrm{In}_{2} \mathrm{Bi}$-Sn system [62] (Fig. 4a), and a transparent alloy [63]. The morphology diagram of two-dimensional ABC and ABAC patterns has been studied in a numericalsimulation study for a model symmetric ternary-eutectic alloy [61]. It was found that both types of three-phased patterns are stable for a finite range of spacing values at given $V$. The upper stability limit of ABAC patterns 
corresponds to a period-preserving oscillatory mode, and the lower one to a lamella elimination (which, in contrast with binary lamellar eutectics, is not directly related to a diffusive process). Experimentally, no stable ABC lamellar patterns have been observed (this motif breaks the right-left symmetry, and may be expected to drift laterally in a real alloy). An experimental study of the stability balloon of ABAC patterns still remains to be completed.

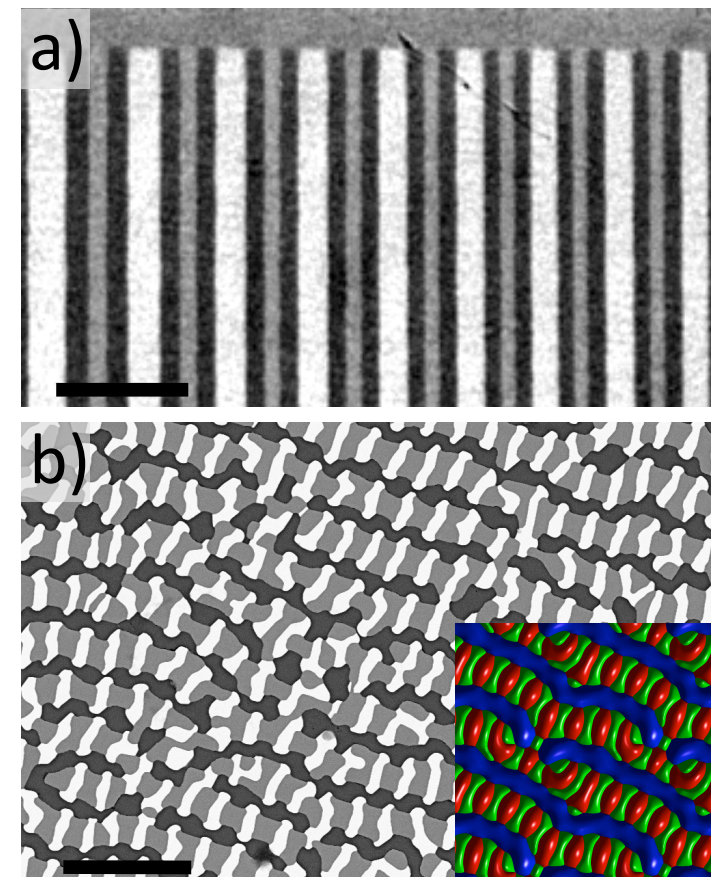

Figure 4: Three-phased growth patterns in directionally solidified nonvariant ternary eutectic alloys. a) Thin $\mathrm{In}_{-} \mathrm{In}_{2} \mathrm{Bi}-\mathrm{Sn}$ alloy; $V=0.05 \mu \mathrm{ms}^{-1}$ (S. Bottin-Rousseau); also see Ref. [62]. b) Bulk Al- $\mathrm{Al}_{2} \mathrm{Cu}-\mathrm{Ag}_{2} \mathrm{Al}$ alloy; $V=0.5 \mu m s^{-1}$ (A. Genau, L. Ratke). Inset: phase-field simulation (A. Choudhury). Bars: $50 \mu m$

In bulk alloys, three-phased growth microstructures most commonly exhibit a marked spatial disorder, and a strong eutectic-grain dependence. In spite of this, three different types (at least) of regular patterns have been clearly identified experimentally. The first one corresponds to the above mentioned ABAC lamellar pattern, and has been observed in bulk solidification of the $\mathrm{In}-\mathrm{In}_{2} \mathrm{Bi}-\mathrm{Sn}$ alloy already mentioned above [64]. The second one presents a regular hexagonal organization, and has been occasionally reported in a transparent alloy [63]. The third one has been called "brick-like pattern", and observed in both $\mathrm{Al}-\mathrm{Al}_{2} \mathrm{Cu}-\mathrm{Ag}_{2} \mathrm{Al}$ (Fig. 4b) and $\mathrm{Al}_{3} \mathrm{Nb}-\mathrm{Nb}_{2} \mathrm{Al}-\mathrm{AlNbNi}$ 
alloys [65, 66]. Brick-like patterns can only be reproduced in phase-field simulations if the three phases have solid-liquid interfacial free energies that differ substantially from each other [67].

\section{Peritectics}

The understanding of solidification patterns in peritectic alloys is considerably less advanced than for eutectics. The reasons are the far greater variety of growth modes that are possible in peritectics, and the fact that steady-state growth is rarely reached. Peritectic alloys exhibit a three-phase equilibrium between two solids and a liquid at the peritectic temperature $T_{p}$. In contrast with eutetics, the liquid contains more solute than both solid phases. Furthermore, there is a stable solid-liquid equilibrium for temperatures above and below $T_{p}$, and a stable solid-solid equilibrium below $T_{p}$. The most frequent solidification path for peritectics is the growth of primary dendrites, followed by nucleation of the peritectic phase. The latter can grow by solidification from the liquid, and by peritectic transformation from the primary solid.

Considerable attention has recently been paid to the two-phase microstructures that form in peritectics during directional solidification for conditions in which both phases are morphologically stable, so that no dendrites can form. Under these conditions, a growth mode called "banding" was proposed [68]: the primary phase grows as a planar front, until the peritectic phase nucleates below $T_{p}$ and spreads laterally over the front. The growth of the peritectic phase, which contains more solute than the primary phase, decreases the solute concentration in the liquid close to the front, the interface temperature rises, and the primary phase can re-nucleate above $T_{p}$. For a narrow band of compositions, this process can repeat and lead to a banding cycle, that is, the alternating growth of layers of the two phases [68]. Another possible growth morphology, namely coupled growth of lamellae or rods like in eutectics, has been discussed already long ago [69] and is now well documented [70]. It has also been shown that during banding the new phases generally nucleate almost simultaneously at different positions along the solid-liquid interface, and that banding can be only partial due to the interactions of the solute diffusion field with walls or the other nuclei ("island banding" [71]), and that it can precede and even prepare coupled growth. The details of the morphology depend not only on the phase diagram, but also on the nucleation parameters (undercooling, frequency) [72]. 
In recent years, two lines of development have added new details to this picture. A series of experiments have been performed on the peritectic alloy $\mathrm{Cu}-\mathrm{Sn}[73,74,75]$. In comparison to the alloys studied previously, $\mathrm{Cu}-\mathrm{Sn}$ has a much larger solidification interval, which implies that extremely low growth rates have to be used to avoid morphological instabilities. In order to reduce solutal convection as much as possible, capillary tubes were used. The microstructures were analyzed using standard metallography, EBSD, as well as post mortem X-ray tomography [74] to obtain a three-dimensional view (see Fig. 5). Alternating regions of one- and two-phase growth were observed. In the two-phase regions, coupled growth in lamellar, rod, or mixed morphology was found, but island morphologies were also obseved. The reasons for the transitions between the different morphologies are not always clear. In addition, the EBSD analysis revealed that a large part of the samples consisted of very few distinct grains. This indicates that the two-phase structures form by morphological instabilities and branching from a small number of nuclei, and not from a large number of independent nuclei. Since unsteady two-phase growth often includes rapid spreading along preexisting interfaces (quite like the "invasion" process documented for eutectics [76]), nucleation may actually not be necessary to produce a structure that looks like bands in a longitudinal cross-section.

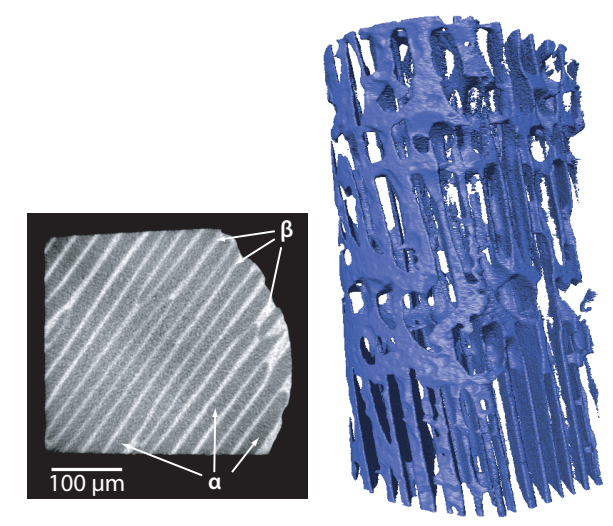

Figure 5: X-ray tomography analysis of coupled growth structures in Sn-Cu (from [74] with permission). The regular lamellar pattern seen in the cross-section (left) is not in steady state: the three-dimensional recostruction shows that short periods of (near) steady-state growth are interrupted by numerous breakup and reconnection events occur.

An oscillatory mode for lamellae in which the thickness of all lamellae of one phase oscillate in register was also observed; this is an instability mode 
that was never observed in eutectics in three dimensions. These oscillations were well reproduced in two-dimensional phase-field simulations carried out with all the parameters matching the experiments [77]. Finally, measurements and phase-field simulations revealed that in the coupled growth mode, the interface temperature is very close to the peritectic temperature, with parts of the interface being above and other parts below $T_{p}$ [77].

The spreading of a "finger" of the peritectic phase on the primary solid phase has also been further investigated. It is generally believed that the primary phase partially remelts before it is covered by the peritectic phase. This scenario was indeed confirmed by phase-field [78] and boundary-integral simulations [79] for generic alloy systems in the one-sided case (no diffusion in the solid), and scaling laws for the amount of remelting and the spreading velocity in isothermal peritectic solidification were obtained [79].

One specific peritectic system deserves special mention: steel. Indeed, there is a peritectic point in the iron-carbon phase diagram at low carbon concentrations. Impressive in situ observations of peritectic spreading in this system have been made using the technique of high-temperature laserscanning confocal microscopy $[80,81]$. Contrary to most of the systems reviewed so far, the diffusivity of the solute (carbon) in liquid and solid is of comparable order of magnitude. Therefore, the peritectic transformation has to be taken into account, even for the study of solidification microstructures. Phase-field simulations of primary ferrite dendrites that are later on covered by the peritectic austenite have been performed already at the end of the 1990's [82]. More recently, the effect of solid-state diffusion around the trijunction point and its influence on the spreading of the peritectic phase has been analyzed [83, 84]. It was found that the presence of appreciable solidstate diffusion greatly reduces the melting of the primary phase and can drastically accelerate the spreading. Finally, as a historic remark, it should be mentioned that the landmark papers of Zener [1] and Hillert [2] on coupled growth morphologies were actually motivated by the study of pearlite growth in steels. This problem continues to be of interest today [85], but its full discussion requires to include effects that are typical of solid-state transformations, such as elastic strains and surface diffusion, which are outside of the scope of the present review. 


\section{Conclusions and outlook}

We have reviewed here recent developments in our understanding of microstructure formation during eutectic and peritectic two-phase growth. Strong focus put on the relation between microstructure formation and the fundamental physics of pattern formation was motivated by our main field of expertise; therefore, many materials-science related aspects have been omitted. However, it should be stressed that aspects of pattern formation and self-organization may be useful for practical applications. For example, it was recently suggested that eutectic growth could be used as a bottom-up fabrication route for advanced optical materials that use micro- and nanostructutres to obtain novel properties (photonic crystals [86], metamaterials [87, 88], plasmonic materials [89]). The success of such strategies requires a combination of deep knowledge about the materials properties and the organization of the patterns.

There are numerous open questions following up on all the lines of research that we have touched upon in the preceding sections. Three areas seem particularly interesting to us for future research: anisotropy effects, irregular eutectics, and peritectics. Concerning anisotropy, we have only begun to understand the complex and intricate relationships between the crystallographic structure of the two-phase composites and the pattern-formation process. What is the effect of anisotropy on pattern selection ? One may anticipate that anisotropy in the azimutal plane (perpendicular to the temperature gradient) will favor the emergence of lamellae over rods. But what is its influence on the pattern dynamics (e.g. the various instability modes, the lamellae-to-rod transition) ? Phase-field simulations [46, 90] seem to be a promising tool to address such questions and need to be extended to three dimension and to more realistic phase diagrams. They also need to be combined with experiments in which the crystallographic orientations can be determined. Furthermore, direct information about the interphase boundary energies would be of the utmost importance. A promising method to access such information are atomistic simulations, along the lines described in the article by Karma and Tourret in this issue. A preliminary simulation of interphase boundaries in Al-Cu is availlable [91], but needs to be extended to higher temperatures and other alloys.

We have not touched, in our review, the subject of irregular eutectics (in which at least one of the phases grows with facetted solid-liquid interfaces). For an example of in situ observations with X-ray techniques, see [92]. Many 
fundamental questions relating to this growth mode remain open. Here again, more detailed information on the structure and kinetics of interfaces coming from atomistic simulations would be extremely helpful. To our knowledge, there is currently no phase-field model that describes the non-linear interface kinetics that are characeteristic for facetted growth.

Finally, many questions on peritectics still remain open. How widespread is the coupled growth morphology? What triggers the transition between morphologies? The numerical simulations of pertectics have so far been limited to two dimensions. What happens to the concept of a "peritectic finger" in three dimensions? How is the primary phase covered after the appearance of the peritectic phase? Can one further elucidate the spreading dynamics under non-stationary conditions ? What is the influence of crystallographic effects on peritectic patterns? These are a few of the many open questions. We believe that many of them can be successfully addressed by pursuing the methodology highlighted in this review: a combination of carefully designed experiments with quantitative numerical simulations. The recent develop-

ments on both aspects show that the potential of these methods remains to be fully exploited.

\section{Acknowledgments}

This work was financially supported by Centre National d'Etudes Spatiales (CNES), France.

[1] C. Zener, Kinetics of the decomposition of Austenite, Trans. AIME 167 (1946) 550-595.

[2] M. Hillert, The role of interfacial energy during solid state phase transformations, Jernkontorets Ann. 141 (1957) 773.

[3] K. A. Jackson, J. D. Hunt, Lamellar and rod eutectic growth, Transactions of the Metallurgical Society of AIME 236 (1966) 1129.

[4] W. Kurz, D. J. Fisher, Fundamentals of Solidification, Trans Tech Publications, Zurich, Switzerland, 1998.

[5] J. A. Dantzig, M. Rappaz, Solidification, EPFL Press, Lausanne, 2009.

[6] H. Kerr, W. Kurz, Solidification of peritectic alloys, INTERNATIONAL MATERIALS REVIEWS 41 (1996) 129-164. 
[7] W. Boettinger, S. Coriell, A. Greer, A. Karma, W. Kurz, M. Rappaz, R. Trivedi, Solidification microstructures: Recent developments, future directions, ACTA MATERIALIA 48 (2000) 43-70. doi:10.1016/S13596454(99)00287-6.

[8] U. Hecht, L. Granasy, T. Pusztai, B. Bottger, M. Apel, V. Witusiewicz, L. Ratke, J. De Wilde, L. Froyen, D. Camel, B. Drevet, G. Faivre, S. Fries, B. Legendre, S. Rex, Multiphase solidification in multicomponent alloys., MATERIALS SCIENCE \& ENGINEERING R-REPORTS 46 (2004) 1-49. doi:10.1016/j.mser.2004.07.002.

[9] A. Karma, M. Plapp, New insights into the morphological stability of eutectic and peritectic coupled growth, JOM 56 (2004) 28-32.

[10] J. Llorca, V. M. Orera, Directionally solidified eutectic ceramic oxides, PROGRESS IN MATERIALS SCIENCE 51 (2006) 711-809. doi:10.1016/j.pmatsci.2005.10.002.

[11] M. Asta, C. Beckermann, A. Karma, W. Kurz, R. Napolitano, M. Plapp, G. Purdy, M. Rappaz, R. Trivedi, Solidification microstructures and solid-state parallels: Recent developments, future directions, ACTA MATERIALIA 57 (2009) 941-971. doi:10.1016/j.actamat.2008.10.020.

[12] N. Provatas, K. Elder, Phase-field methods in materials science and engineering, Wiley-VCH, Weinheim, 2010.

[13] M. Plapp, Phase-field models, in: T. Nishinaga (Ed.), The Handbook of Crystal Growth, 2nd edition, Vol. 1B, Elsevier, Amsterdam, 2015, pp. 631-668.

[14] A. Parisi, M. Plapp, Stability of lamellar eutectic growth, Acta Materialia 56 (2008) 1348.

[15] A. Parisi, M. Plapp, Defects and multistability in eutectic solidification patterns, EPL 90 (2010) 26010.

[16] M. Perrut, A. Parisi, S. Akamatsu, S. Bottin-Rousseau, G. Faivre, M. Plapp, Role of transverse temperature gradients in the generation of lamellar eutectic solidification patterns, Acta Mat. 58 (2010) 17611769. 
[17] S. Bottin-Rousseau, M. Perrut, C. Picard, S. Akamatsu, G. Faivre, An experimental method for the in situ observation of eutectic growth patterns in bulk samples of transparent alloys, Journal of Crystal Growth 306 (2007) 465-472.

[18] V. T. Witusiewicz, U. Hecht, S. Rex, Top-view approach for insitu observation of growth morphology in bulk transparent organic alloys, JOURNAL OF CRYSTAL GROWTH 353 (2012) 17-24. doi:10.1016/j.jcrysgro.2012.04.040.

[19] H. Nguyen-Thi, L. Salvo, R. H. Mathiesen, L. Arnberg, B. Billia, M. Suery, G. Reinhart, On the interest of synchrotron X-ray imaging for the study of solidification in metallic alloys, COMPTES RENDUS PHYSIQUE 13 (2012) 237-245. doi:10.1016/j.crhy.2011.11.010.

[20] J. S. Langer, Instabilities and pattern formation in crystal growth, Rev. Mod. Phys. 52 (1) (1980) 1.

[21] K. Kassner, C. Misbah, Growth of lamellar eutectic structures: The axisymmetric case, Phys. Rev. A 44 (10) (1991) 6513.

[22] K. Kassner, C. Misbah, Similarity laws in eutectic growth, Phys. Rev. Lett. 66 (1991) 445-448.

[23] P. Manneville, Dissipative Structures and Weak Turbulence, Academic Press, Boston, 1990.

[24] M. C. Cross, P. C. Hohenberg, Pattern formation outside of equilibrium, Rev. Mod. Phys. 65 (1993) 851.

[25] M. Ginibre, S. Akamatsu, G. Faivre, Experimental determination of the stability diagram of a lamellar eutectic growth front, Phys. Rev. E 56 (1) (1997) 780 .

[26] A. Karma, A. Sarkissian, Morphological instabilities of lamellar eutectics, Metall. Mat. Trans. A 27 (1996) 635.

[27] S. Akamatsu, M. Plapp, G. Faivre, A. Karma, Overstability of lamellar eutectic growth below the minimum-undercooling spacing, Metall. Mat. Trans. A 35 (2004) 1815. 
[28] R. Folch, M. Plapp, Quantitative phase-field modeling of two-phase solidification, Phys. Rev. E 72 (1) (2005) 011602.

[29] S. Akamatsu, S. Bottin-Rousseau, G. Faivre, Experimental evidence for a zigzag bifurcation in bulk lamellar eutectic growth, Phys. Rev. Lett. 93 (2004) 175701.

[30] S. Akamatsu, S. Bottin-Rousseau, G. Faivre, Stability of lamellar eutectic growth in thick samples, Philosophical Magazine 86 (24) (2006) 3703 .

[31] S. Liu, J. H. Lee, R. Trivedi, Dynamic effects in the lamellarrod eutectic transition, ACTA MATERIALIA 59 (2011) 3102-3115. doi:10.1016/j.actamat.2011.01.050.

[32] H. Walker, S. Liu, J. H. Lee, R. Trivedi, Eutectic growth in three dimensions, Met. Mat. Trans. A 38A (7) (2007) 1417-1425.

[33] U. Hecht, V. Witusiewicz, A. Drevermann, S. Rex, Orientation relationship in univariant $\mathrm{Al}-\mathrm{Cu}-\mathrm{Ag}$ eutectics, Trans. Indian Inst. Met. 58 (2005) 545-551.

[34] M. Perrut, S. Akamatsu, S. Bottin-Rousseau, G. Faivre, Long-time dynamics of the directional solidification of rodlike eutectics, Phys. Rev. E 79 (2009) 032602.

[35] M. Perrut, S. Bottin-Rousseau, G. Faivre, S. Akamatsu, Dynamic instabilities of rod-like eutectic growth patterns: A real-time study, ACTA MATERIALIA 61 (2013) 6802-6808. doi:10.1016/j.actamat.2013.07.054.

[36] V. Trnovcová, P. P. Fedorov, C. Bàrta, V. Labas, V. A. Meleshina, B. P. Sobolev, Microstructure and physical properties of superionic eutectic composites of the lifrf3 (r 5 rare earth element) system, Solid State Ionics 119 (1999) 173-180.

[37] R. E. Napolitano, M. Serefoglu, Control and Interpretation of FiniteSize Effects and Initial Morphology in Directional Solidification of a Rod-Type Eutectic Transparent Metal-Analog, JOM 64 (2012) 68-75. doi:10.1007/s11837-011-0214-z. 
[38] M. Plapp, M. Dejmek, Stability of hexagonal solidification patterns, Europhys. Lett. 65 (2004) 276.

[39] N. Bergeon, D. Tourret, L. Chen, J.-M. Debierre, R. Guérin, A. Ramirez, B. Billia, A. Karma, R. Trivedi, Spatiotemporal dynamics of oscillatory cellular patterns in three-dimensional directional solidification, Phys. Rev. Lett. 110 (2013) 226102. doi:10.1103/PhysRevLett.110.226102.

[40] R. Racek, G. Lesoult, M. Turpin, Cd-Sn Eutectic Structures at Low Growth-rates, J. Cryst. Growth 22 (1974) 210-218. doi:10.1016/00220248(74)90096-7.

[41] A. Ludwig, J. Mogeritsch, M. Kolbe, G. Zimmermann, L. Sturz, N. Bergeon, B. Billia, G. Faivre, S. Akamatsu, S. Bottin-Rousseau, D. Voss, Advanced Solidification Studies on Transparent Alloy Systems: A New European Solidification Insert for Material Science Glovebox on Board the International Space Station, JOM 64 (2012) 1097-1101. doi:10.1007/s11837-012-0403-4.

[42] L. Ratke, J. Alkemper, Ordering of the fibrous eutectic microstructure of Al-Al3Ni due to accelerated solidification conditions, ACTA MATERIALIA 48 (2000) 1939-1948. doi:10.1016/S1359-6454(99)00461-9.

[43] L. Hogan, R. Kraft, F. Lemkey, Eutectic grains, Adv. Mater. Res. 5 (1971) 83-216.

[44] S. Akamatsu, S. Bottin-Rousseau, M. Şerefoğlu, G. Faivre, A theory of thin lamellar eutectic growth with anisotropic interphase boundaries, Acta Materialia 60 (2012) 3199-3205.

[45] S. Akamatsu, S. Bottin-Rousseau, M. Şerefoğlu, G. Faivre, lamellar eutectic growth with anisotropic interphase boundaries: Experimental study using rotational directional solidification, Acta Materialia 60 (2012) 3206-3214.

[46] S. Ghosh, A. Choudhury, M. Plapp, S. Bottin-Rousseau, G. Faivre, S. Akamatsu, Interphase anisotropy effects on lamellar eutectics: A numerical study, PHYSICAL REVIEW E 91. doi:10.1103/PhysRevE.91.022407. 
[47] C. T. Rios, S. Milenkovic, R. Caram, A novel ternary eutectic in the nbalni system, Scripta Materialia 48 (2003) 1495-1500.

[48] M. Rinaldi, R. Sharp, M. Flemings, Growth of ternary composites from melt, Metall. Trans. 3 (1972) 3133.

[49] M. Plapp, A. Karma, Eutectic colony formation: A stability analysis, Phys. Rev. E 60 (1999) 6865-6889.

[50] S. Akamatsu, G. Faivre, Traveling waves, two-phase fingers, and eutectic colonies in thin-sample directional solidification of a ternary eutectic alloy, Phys. Rev. E 61 (2000) 3757-3770.

[51] M. Plapp, A. Karma, Eutectic colony formation: A phase-field study, Phys. Rev. E 66 (2002) 061608.

[52] Z. Shang, J. Shen, J. Zhang, L. Wang, L. Wang, H. Fu, Effect of microstructures on the room temperature fracture toughness of NiAl-32Cr-6Mo hypereutectic alloy directionally solidified at different withdrawal rates, MATERIALS SCIENCE AND ENGINEERING A-STRUCTURAL MATERIALS PROPERTIES MICROSTRUCTURE AND PROCESSING 611 (2014) 306-312. doi:10.1016/j.msea.2014.05.090.

[53] P. Fehrenbach, H. Kerr, P. Niessen, UNIDIRECTIONAL SOLIDIFICATION OF MONOVARIANT CU-NI-MG EUTECTIC ALLOYS .2. MICROSTRUCTURES AND PROPERTIES, JOURNAL OF CRYSTAL GROWTH 18 (1973) 151-158.

[54] S. Tewari, S. Raj, I. Locci, A comparison between growth morphology of "eutectic" cells/dendrites and single-phase cells/dendrites, METALLURGICAL AND MATERIALS TRANSACTIONS A-PHYSICAL METALLURGY AND MATERIALS SCIENCE 35A (2004) 1632-1635. doi:10.1007/s11661-004-0269-3.

[55] S. Akamatsu, M. Perrut, S. Bottin-Rousseau, G. Faivre, Spiral Two-Phase Dendrites, PHYSICAL REVIEW LETTERS 104. doi:10.1103/PhysRevLett.104.056101. 
[56] S. Akamatsu, S. Bottin-Rousseau, G. Faivre, E. A. Brener, Scaling Theory of Two-Phase Dendritic Growth in Undercooled Ternary Melts, PHYSICAL REVIEW LETTERS 112. doi:10.1103/PhysRevLett.112.105502.

[57] T. Pusztai, L. Ratkai, A. Szallas, L. Granasy, Spiraling eutectic dendrites, PHYSICAL REVIEW E 87. doi:10.1103/PhysRevE.87.032401.

[58] L. Rátkai, A. Szállás, T. Pusztai, T. Mohri, L. Gránásy, Ternary eutectic dendrites: Pattern formation and scaling properties, J. Chem. Phys. (2015) 1.

[59] G. Boussinot, C. Hueter, E. A. Brener, Growth of a two-phase finger in eutectics systems, Phys. Rev. E 83. doi:10.1103/PhysRevE.83.020601.

[60] T. Himemiya, T. Umeda, Three-phase planar eutectic growth models for a ternary eutectic system, MATERIALS TRANSACTIONS JIM 40 (1999) 665-674.

[61] A. Choudhury, M. Plapp, B. Nestler, Theoretical and numerical study of lamellar eutectic three-phase growth in ternary alloys, PHYSICAL REVIEW E 83. doi:10.1103/PhysRevE.83.051608.

[62] V. Witusiewicz, U. Hecht, S. Rex, M. Apel, In situ observation of microstructure evolution in low-melting Bi-In-Sn alloys by light microscopy, ACTA MATERIALIA 53 (2005) 3663-3669. doi:10.1016/j.actamat.2005.04.015.

[63] L. Sturz, V. Witusiewicz, U. Hecht, S. Rex, Organic alloy systems suitable for the investigation of regular binary and ternary eutectic growth, JOURNAL OF CRYSTAL GROWTH 270 (2004) 273-282. doi:10.1016/j.jcrysgro.2004.06.004.

[64] M. Ruggiero, J. Rutter, Origin of microstructure in the $332 \mathrm{~K}$ eutectic of the Bi-In-Sn system, MATERIALS SCIENCE AND TECHNOLOGY 13 (1997) $5-11$.

[65] A. Genau, L. Ratke, Morphological characterization of the Al-Ag-Cu ternary eutectic, INTERNATIONAL JOURNAL OF MATERIALS RESEARCH 103 (2012) 469-475. doi:10.3139/146.110652. 
[66] R. J. Contieri, C. T. Rios, M. Zanotello, R. Caram, Growth and three-dimensional analysis of a Nb-Al-Ni ternary eutectic, MATERIALS CHARACTERIZATION 59 (2008) 693-699. doi:10.1016/j.matchar.2007.05.025.

[67] A. Choudhury, A. Dennstedt, L. Ratke, B. Nestler, Role of composition and solid-liquid surface energy in self-organization of phases during three-phase eutectic growth, unpublished (2015).

[68] R. Trivedi, THEORY OF LAYERED-STRUCTURE FORMATION IN PERITECTIC SYSTEMS, METALLURGICAL AND MATERIALS TRANSACTIONS A-PHYSICAL METALLURGY AND MATERIALS SCIENCE 26 (1995) 1583-1590. doi:10.1007/BF02647608.

[69] W. J. Boettinger, Structure of directionally solidified 2-phase SnCd Peritectic alloys, Metallurgical Transactions 5 (1974) 2023-2031. doi:10.1007/BF02644495.

[70] S. Dobler, T. S. Lo, M. Plapp, A. Karma, Peritectic coupled growth, Acta Mat. 52 (9) (2004) 2795.

[71] T. Lo, S. Dobler, M. Plapp, A. Karma, W. Kurz, Two-phase microstructure selection in peritectic solidification: from island banding to coupled growth, ACTA MATERIALIA 51 (2003) 599-611. doi:10.1016/S13596454(02)00440-8.

[72] T. Lo, A. Karma, M. Plapp, Phase-field modeling of microstructural pattern formation during directional solidification of peritectic alloys without morphological instability, Phys. Rev. E 63. doi:10.1103/PhysRevE.63.031504.

[73] F. Kohler, L. Germond, J. D. Wagniere, M. Rappaz, Peritectic solidification of Cu-Sn alloys: Microstructural competition at low speed, ACTA MATERIALIA 57 (2009) 56-68. doi:10.1016/j.actamat.2008.08.058.

[74] M. Rappaz, F. Kohler, J. Valloton, A. B. Phillion, M. Stampanoni, Connectivity of Phases and Growth Mechanisms in Peritectic Alloys Solidified at Low Speed: an X-Ray Tomography Study of Cu-Sn, METALLURGICAL AND MATERIALS TRANSACTIONS A-PHYSICAL METALLURGY AND MATERIALS SCIENCE 41A (2010) 563-567. doi:10.1007/s11661-009-0118-5. 
[75] J. Valloton, J. D. Wagniere, M. Rappaz, Competition of the primary and peritectic phases in hypoperitectic $\mathrm{Cu}-\mathrm{Sn}$ alloys solidified at low speed in a diffusive regime, ACTA MATERIALIA 60 (2012) 3840-3848. doi:10.1016/j.actamat.2012.03.030.

[76] S. Akamatsu, S. Moulinet, G. Faivre, The formation of lamellareutectic grains in thin samples, METALLURGICAL AND MATERIALS TRANSACTIONS A-PHYSICAL METALLURGY AND MATERIALS SCIENCE 32 (2001) 2039-2048. doi:10.1007/s11661-001-0016-y.

[77] J. Valloton, J. A. Dantzig, M. Plapp, M. Rappaz, Modeling of peritectic coupled growth in Cu-Sn alloys, ACTA MATERIALIA 61 (2013) 55495560. doi:10.1016/j.actamat.2013.05.011.

[78] G. Boussinot, E. A. Brener, D. E. Temkin, Kinetics of isothermal phase transformations above and below the peritectic temperature: Phase-field simulations, ACTA MATERIALIA 58 (2010) 1750-1760. doi:10.1016/j.actamat.2009.11.017.

[79] G. Boussinot, C. Hueter, R. Spatschek, E. A. Brener, Isothermal solidification in peritectic systems, ACTA MATERIALIA 75 (2014) 212-218. doi:10.1016/j.actamat.2014.04.055.

[80] H. Shibata, Y. Arai, M. Suzuki, T. Emi, Kinetics of peritectic reaction and transformation in Fe-C alloys, Met. Mat. Trans. B 31 (2000) 981991, Geoffrey Belton Memorial Symposium, SYDNEY, AUSTRALIA, JAN, 2000. doi:10.1007/s11663-000-0074-3.

[81] D. Phelan, M. Reid, R. Dippenaar, Kinetics of the peritectic reaction in an Fe-C alloy, MATERIALS SCIENCE AND ENGINEERING A-STRUCTURAL MATERIALS PROPERTIES MICROSTRUCTURE AND PROCESSING 477 (2008) 226-232. doi:10.1016/j.msea.2007.05.090.

[82] J. Tiaden, Phase field simulations of the peritectic solidification of Fe-C, JOURNAL OF CRYSTAL GROWTH 198 (1999) 1275-1280, 12th International Conference on Crystal Growth, held in Conjunction with the 10th International Conference on Vapor Growth and Epitaxy (ICCG-12/ICVGE-10), JERUSALEM, ISRAEL, JUL 26-31, 1998. doi:10.1016/S0022-0248(98)01009-4. 
[83] M. Ohno, K. Matsuura, Diffusion-controlled peritectic reaction process in carbon steel analyzed by quantitative phasefield simulation, ACTA MATERIALIA 58 (2010) 6134-6141. doi:10.1016/j.actamat.2010.07.031.

[84] M. Ohno, K. Matsuura, Motion and Morphology of Triple Junction in Peritectic Reaction Analyzed by Quantitative Phasefield Model, ISIJ INTERNATIONAL $50 \quad$ (2010) 1879-1885. doi:10.2355/isijinternational.50.1879.

[85] I. Steinbach, M. Apel, The influence of lattice strain on pearlite formation in fe-c, Acta Mater. 55 (2007) 4817.

[86] D. Pawlak, G. Lerondel, I. Dmytruk, Y. Kagamitani, S. Durbin, P. Royer, T. Fukuda, Second order self-organized pattern of terbiumscandium-aluminum garnet and terbium-scandium perovskite eutectic, JOURNAL OF APPLIED PHYSICS 91 (2002) 9731-9736. doi:10.1063/1.1479752.

[87] D. Pawlak, K. Kolodziejak, S. Turczynski, J. Kisielewski, K. Rozniatowski, R. Diduszko, M. Kaczkan, M. Malinowski, Self-organized, rodlike, micrometer-scale microstructure of Tb3Sc2Al3O12-TbSCO3 : Pr eutectic, CHEMISTRY OF MATERIALS 18 (2006) 2450-2457. doi:10.1021/cm060136h.

[88] D. A. Pawlak, S. Turczynski, M. Gajc, K. Kolodziejak, R. Diduszko, K. Rozniatowski, J. Smalc, I. Vendik, How Far Are We from Making Metamaterials by Self-Organization? The Microstructure of Highly Anisotropic Particles with an SRR-Like Geometry, ADVANCED FUNCTIONAL MATERIALS 20 (2010) 1116-1124. doi:10.1002/adfm.200901875.

[89] K. Sadecka, M. Gajc, K. Orlinski, H. B. Surma, A. Klos, I. Jozwik-Biala, K. Sobczak, P. Dluzewski, J. Toudert, D. A. Pawlak, When Eutectics Meet Plasmonics: Nanoplasmonic, Volumetric, Self-Organized, SilverBased Eutectic, ADVANCED OPTICAL MATERIALS 3 (2015) 381389. doi:10.1002/adom.201400425.

[90] A. Choudhury, M. Geeta, B. Nestler, Influence of solid-solid interface anisotropy on three-phase eutectic growth during directional solidification, EPL 101. doi:10.1209/0295-5075/101/26001. 
[91] V. Kokotin, U. Hecht, Molecular dynamics simulations of Al-Al2Cu phase boundaries, Computational Materials Science 86 (2014) 30-37. doi:10.1016/j.commatsci.2014.01.014.

[92] C. M. Gourlay, K. Nogita, A. K. Dahle, Y. Yamamoto, K. Uesugi, T. Nagira, M. Yoshiya, H. Yasuda, In situ investigation of unidirectional solidification in $\mathrm{Sn}-0.7 \mathrm{Cu}$ and $\mathrm{Sn}-0.7 \mathrm{Cu}-0.06 \mathrm{Ni}$, ACTA MATERIALIA 59 (2011) 4043-4054. doi:10.1016/j.actamat.2011.03.028. 